\title{
Opening the doors of perception in the irritable bowel syndrome
}

It is now nearly 25 years since Ritchie ${ }^{1}$ first reported that compared with control subjects, patients with irritable bowel syndrome (IBS) exhibit decreased pain thresholds to balloon distension of the pelvic colon. However, the concept that disordered gastrointestinal perception might have a significant pathophysiological role in IBS has only become fashionable during the past decade.

Gastrointestinal sensitivity can be assessed using a number of different stimuli, but the one most commonly used is balloon distension, which can be applied to the gut progressively either in the form of volume or pressure increments (ascending series). The latter involves the use of a barostat which is a computer controlled pump able to inject or withdraw air rapidly from the balloon, thus maintaining a constant pressure irrespective of changes in contractility or tone of the area concerned. In most areas of the gut, a measure of visceral perception is taken as the distension threshold (volume or pressure) to induce discomfort; however, in the rectum, similar thresholds can be obtained for the sensations of gas, call to stool and urgency.

There are a number of technical points that have to be taken into consideration when making comparisons between laboratories. These include whether the distension is applied in a ramp (steady increase), intermittent (returning to baseline between inflations) or stepwise (incremental steps in distension) manner, with even the rate of inflation affecting results-low sensory thresholds tend to be elicited in patients with IBS by rapid rather than by slow rectal distension. ${ }^{2}$ In addition, there is some controversy about the definition of sensory thresholds with some investigators choosing to use the descriptor "pain" and others "discomfort". Furthermore, many of the older papers on this subject have compared groups in terms of a mean and range of values and only recently has there been a trend towards calculating the percentage number of patients outside a defined normal range.

Despite all these difficulties, most studies have shown that approximately $60 \%$ of hospital based patients with IBS have lowered thresholds to rectal distension, ${ }^{34}$ although up to $94 \%$ of patients can be classified as having abnormal visceral perception if two other sensory indicators are included. ${ }^{4}$ These are the way the intensity of the discomfort is rated by the patient and the extent of its viscerosomatic referral. This has lead Mertz and colleagues to propose that altered rectal perception may be a biological marker for IBS. ${ }^{4}$

The pathophysiological mechanisms responsible for altered visceral perception thresholds remain unclear. Abnormal compliance of the gut does not seem to be a major factor, ${ }^{15-7}$ and there is currently considerable interest in psychological influences on perception. It has been suggested recently that the hypersensitivity shown in patients with IBS may, in part, reflect a tendency by the patient to anticipate a noxious stimulus rather than them being truely hypersensitive to it - the so called perceptual response bias. This problem can to some extent be overcome by the use of "tracking" or "double random staircase" isobaric distension techniques, in which distension stimuli are applied to the gut in an unpredictable way, and results obtained using this method tend to support the response bias hypothesis.

In this issue (see page 505) the UCLA group have sought to characterise further the perceptual alterations to rectal stimuli in IBS by comparing the thresholds obtained between patients and controls using traditional and tracking distension techniques coupled with the way the subject reported the stimulus in terms of intensity (sensory scale) and unpleasantness (affective scale). They have shown that although the discomfort thresholds for the ascending series were significantly lower for the group of patients with IBS compared with controls, the discomfort thresholds during the tracking procedure did not differ between groups. However, when the tracking data were examined on an individual basis, they were able to show that nearly half the patients exhibited hypersensitivity as reflected by discomfort thresholds below the $95 \%$ confidence interval of that for the mean of the controls, together with a left-shift of their intensity (sensory) and unpleasantness (affective) stimulus response curves. The fact that a substantial proportion of their patients can be classified as hypersensitive, despite there being no differences in overall group comparisons, raise some concerns about the composition of the patient group which cannot be answered from the information given in the paper. Furthermore, the derivation of their normal ranges is somewhat unconventional. Finally, Naliboff and colleagues have introduced the term hypervigilance to explain the tendency of patients to describe a wide range of visceral stimuli in a rather negative manner. Thus, they suggest there are two overlapping perceptual alterations to rectal distension in patients with IBS - firstly, true hypersensitivity and, secondly, hypervigilance to visceral stimuli.

Why some patients, but not others, should have abnormal thresholds to intraluminal distension is not understood, but it may depend on factors such as regional and/or temporal variations in gut sensitivity. We have found evidence for the former, in that patients who do not have rectal hypersensitivity can have hypersensitivity elsewhere in the gastrointestinal tract, for instance in either the small bowel or colon. ${ }^{7}$ When considering temporal changes in sensitivity, Naliboff et al suggest that during periods of internal and external stress, hypersensitivity could be unmasked. They postulate that the increased sigmoid contractility seen in patients with IBS in response to food $^{8}$ and emotional stress $^{9}$ may sensitise the gut, supporting this idea with their recent observations that high pressure stimulation of the colon sensitises the rectum in patients with IBS but not controls. ${ }^{10}$

What of the future? It would be of interest to know whether any of these perceptual or physiological characteristics observed in patients with IBS attending specialised centres are shared by their counterparts in the community who are never referred to hospital. In addition, much more 
needs to be known about how the gut becomes sensitised (eg luminal events such as inflammation and infection), at what level sensitisation can take place (eg tissue, spinal or central) and whether this is under any form of genetic control. By better defining the mechanisms involved, more rational approaches to treatment may be developed and appropriately targeted. For example, a more hypervigilant patient may respond better to a psychological approach, whereas a truely hypersensitive patient may derive benefit from some form of specific pharmacological intervention.

Lastly, the notion that IBS might be a motility disorder has almost become regarded as rather passé. It may be a little premature to reject the contribution of contractility to the symptoms of IBS just yet, even if it is only a case of a normal contraction being felt more readily because of a perceptual abnormality.

LESLEY A HOUGHTON

Department of Medicine,

University Hospital of South Manchester,

Nell Lane, West Didsbury,

Manchester M20 2LR, UK
1 Ritchie J. Pain from distension of the pelvic colon by inflating a balloon in the irritable colon syndrome. Gut 1973; 14: 125-32.

2 Lembo T, Munakata J, Mertz H, Niazi N, Kodner A, Nikas N, et al. Evidence for the hyperalgesia of lumbar splanchnic afferents in irritable bowel syndrome. Gastroenterology 1994; 107: 1686-96.

3 Prior A, Sorial E, Sun WM, Read NW. Irritable bowel syndrome: differences between patients who show rectal sensitivity and those who do not. Eur $\mathcal{F}$ Gastroenterol Hepatol 1993; 5: 343-9.

4 Mertz H, Naliboff B, Munakata J, Mazi N, Mayer EA. Altered rectal perception is a biological marker of patients with irritable bowel syndrome. Gastroenterology 1995; 109: 40-52.

5 Whitehead WE, Holtkotter B, Enck P, Hoelzl R, Holmes KD, Anthony J, et al. Tolerance for rectosigmoid distension in irritable bowel syndrome. Gastroenterology 1990; 98: 1187-92.

6 Bradette M, Delvaux M, Staumont G, Fioramonti J, Bueno L, Frexinos J. Evaluation of colonic sensory thresholds in IBS patients using a barostat. Definition of optimal conditions and comparison with healthy subjects. Dig Definition of optimal con
Dis Sci 1994; 39: 449-57.

7 Francis CY, Houghton LA, Whorwell PJ, Morris J. Enhanced sensitivity of the whole gut in patients with irritable bowel syndrome [abstract]. Gastroenterology 1995; 108: A601.

8 Bazzocchi G, Ellis J, Villanueva-Meyer J, Reddy SN, Mena I, Snape WJ Jr. Effect of eating on colonic motility and transit in patients with functional diarrhea. Gastroenterology 1991; 101: 1298-306.

9 Welgan P, Meshkinpour H, Hoehler F. The effect of stress on colon motor and electrical activity in irritable bowel syndrome. Psychosom Med 1985; 47: 139-49.

10 Munakata J, Naliboff B, Harraf F, Kodner A, Lembo T, Chang L, et al. Repetitive sigmoid stimulation induces rectal hyperalgesia in patients with irritable bowel syndrome. Gastroenterology 1997; 112: 55-63. 